\title{
Pirólisis de hemicelulosa catalizada por sulfato de zinc y sulfato férrico
}

\section{Hemicellulose pyrolysis catalyzed by zinc sulfate and ferric sulfate}

\author{
Pirólise de hemicelulose catalisada por \\ sulfato de zinco e sulfato férrico
}

\author{
Alberto Albis $^{1 *}$; Éver Ortiz²; Ismael Piñeres²; Juan Osorio ${ }^{1}$; Jennifer Monsalvo ${ }^{1}$ \\ ${ }^{1}$ Facultad de Ingeniería, 2Facultad de Ciencias Básicas, Universidad del Atlántico, km 7 antigua vía a Puerto \\ Colombia, Puerto Colombia, Colombia. \\ *albertoalbis@uniatlantico.edu.co
}

Fecha recepción: 29 de mayo de 2017 Fecha aceptación: 10 de septiembre de 2018

\begin{abstract}
Resumen
Recurriendo al uso de la técnica análisis termogravimétrico, se estudió el efecto catalítico del sulfato férrico y el sulfato de zinc sobre la pirólisis de la hemicelulosa. Se presentan termogramas de pirólisis de la hemicelulosa pura y en mezcla con estos catalizadores, en concentración de $3 \%$ en peso, a las velocidades de calentamiento 10,30 y $100 \mathrm{~K} / \mathrm{min}$. Se analizó el efecto de los catalizadores y de la velocidad de calentamiento sobre la forma de los termogramas, la temperatura de pico DTG de los eventos de pérdida de peso y los parámetros cinéticos del proceso. Los resultados fueron ajustados a tres modelos cinéticos diferentes. La presencia de estas sales cambia considerablemente las formas de los termogramas y la temperatura de los picos DTG, con respecto a los correspondientes termogramas de la hemicelulosa pura. Los datos de los tres sistemas fueron bien modelados por el modelo de distribución de energías de activación, DAEM. A partir de la comparación de estos y los respectivos resultados del modelo de descomposición propuesto en la literatura, es posible inferir que los sulfatos objeto de estudio catalizan las reacciones de deshidratación/fragmentación y depolimerización de la hemicelulosa.
\end{abstract}

Palabras clave: hemicelulosa, pirólisis, sulfato férrico, sulfato de zinc, catálisis.

\begin{abstract}
Using the thermogravimetric analysis technique, the catalytic effect of ferric sulfate and zinc sulfate on the pyrolysis of hemicellulose was studied. Thermograms of the pyrolysis of pure hemicellulose and hemicellulose mixed with $3 \%$ of catalysts were obtained at heating rates of 10,30 , and $100 \mathrm{~K} / \mathrm{min}$. The effect of heating rate and catalysts on the shape of thermograms, DTG peak temperature of weight loss events, and kinetics parameters was evaluated. Results were fitted to three kinetic models. The presence of these salts strongly change the thermogram forms and DTG temperature peaks with respect to the corresponding pure hemicellulose thermograms. Data from the three systems studied were well-modeled by the distributed activation energy model, DAEM. By comparing these and the respective results of the decomposition model proposed in the literature, it is possible to infer that the sulphates under study catalyze the reactions of dehydration/fragmentation and de-polymerization of hemicellulose.
\end{abstract}

Keywords: hemicellulose, pyrolysis, ferric sulfate, zinc sulfate, catalysis.

Cita: Albis A, Ortíz E, Piñeres I, Osorio J, Monsalvo J. Pirólisis de hemicelulosa catalizada por sulfato de zinc y sulfato férrico. rev.ion. 2018;31(2):37-49. doi:10.18273/revion.v31n2-2018003 


\section{Resumo}

Recorrendo à utilização da técnica de análise termogravimétrica, foi estudado o efeito catalítico de sulfato férrico e sulfato de zinco sobre a pirólise de hemicelulose. Os termogramas de pirólise de hemicelulose pura e misturada com estes catalisadores foram obtidos na concentração de $3 \%$ em peso em velocidades de aquecimento de 10, 30 e $100 \mathrm{~K} / \mathrm{min}$. O efeito dos catalisadores e a taxa de aquecimento sobre a forma de termogramas, a temperatura de pico DTG de eventos de perda de peso e os parâmetros cinéticos do processo foram analisados. Os resultados foram ajustados para três modelos cinéticos diferentes. A presença desses sais muda grandemente as formas de termogramas e a temperatura de pico DTG em relação à os correspondentes termogramas de hemicelulose puros. Os dados dos três sistemas foram bem modelados pelo padrão de distribuição de energias de ativação, DAEM. A partir da comparação destes e os respectivos resultados do modelo de decomposição proposto na literatura, pode-se inferir que os sulfatos objeto de estudo catalisam reações de desidratação/fragmentação e despolimerização de hemicelulose.

Palavras-chave: hemicellulose, pirólise, sulfato férrico, sulfato de zinco, catálise.

\section{Introducción}

Por razones de seguridad energética y por el alto costo económico y ambiental que implica el uso de combustibles fósiles, inmensos esfuerzos financieros, científicos y tecnológicos se han direccionado hacia la búsqueda de fuentes alternativas de energía. En países desarrollados y en vía de desarrollo, las fluctuaciones del precio de los combustibles fósiles y el cambio climático han originado un creciente interés en los biocombustibles. En cuanto al uso de mezclas de combustibles fósiles con biocombustibles, la mayoría de los países están cambiando la legislación de una condición voluntaria a obligatoria, especialmente para el caso del sector transporte. Estas políticas podrían contribuir a reducir el cambio climático y a aumentar la seguridad energética. Un valor agregado de este tipo de legislaciones es la diversificación de la producción agrícola. De esta manera, los biocombustibles generan oportunidades al aumentar el ingreso per cápita rural y posiblemente al permitir el acceso a energía comercial. Por estas razones, el sector académico trabaja intensivamente en la búsqueda de recursos de biomasa y en su procesamiento para lograr mayor eficiencia energética en la mezcla de combustibles fósiles con biocombustibles, y de esta forma disminuir las emisiones de efecto invernadero. Ciertamente, los productos de la pirólisis de biomasa (bio-oil) reemplazan los combustibles líquidos sin aumentar la cantidad de $\mathrm{CO}_{2}$ cargada a la atmósfera, puesto que la misma cantidad de este gas es capturada en su proceso de crecimiento. Los principales recursos bioenergéticos disponibles para los procesos pirolíticos son madera, cultivos agrícolas, residuos de cosechas y plantaciones forestales, componentes biodegradables de residuos sólidos municipales y residuos sólidos industriales [1]. Los países latinoamericanos tienen un enorme potencial de recursos bioenergéticos, principalmente en los residuos del sector agroindustrial; sin embargo, estos recursos son bastante heterogéneos y escasamente caracterizados. Algunos estudios [2-4] muestran que efectivamente existe una relación entre la composición de la biomasa y la distribución de los productos de la pirólisis y las propiedades del bio-oil obtenido, por tanto, cada posible materia prima para la pirólisis debe ser sistemáticamente estudiada [5].

Para caracterizar el comportamiento de la pirólisis de la biomasa, se ha optado por investigar inicialmente la pirólisis de cada uno de sus tres principales componentes: celulosa, hemicelulosa y lignina. Las técnicas de caracterización típicamente usadas incluyen análisis termogravimétrico (TG), análisis simultáneo de termogravimetría y cromatografía de gases (TG-GC) y análisis simultáneo de termogravimetría y espectroscopía infrarroja con transformada de Fourier (TG-FTIR) $[6,7]$. Usualmente en etapas más adelantadas de las investigaciones (recurriendo a estas mismas técnicas) se han estudiado las interacciones que ocurren entre los indicados componentes, como también las interacciones que tienen lugar entre cada uno de estos con algunos minerales alcalinos, minerales alcalinotérreos [8] y sales metálicas, particularmente cloruros de zinc y de níquel [9]. En general, los estudios de pirólisis de diferentes tipos de biomasa muestran que el bio-oil obtenido no tiene la calidad suficiente para ser usado directamente como combustible (entre otras dificultades) y, por tanto, es necesario someterlo 
a un proceso de upgrading, lo cual aumenta considerablemente los costos de producción. Un método que mejora la calidad del bio-oil y a su vez disminuye los costos de producción mencionados es la pirólisis catalítica. Sin embargo, para la mayoría de los catalizadores estudiados se reporta una significativa disminución en el rendimiento del producto líquido [10]. Para determinar el impacto que tiene cada uno de estos catalizadores sobre los diferentes tipos de biomasa es necesario primeramente estudiar su efecto sobre cada uno de sus principales constituyentes. El Zn y el Fe son elementos que se pueden encontrar en cantidades apreciables en materiales lignocelulósicos. Se reportó que el sulfato ferroso cambia la distribución de productos en la pirólisis de madera de algodón y papel periódico, disminuye la temperatura de pirólisis y aumenta la producción de levoglucosano y levoglucosanona [11]. Resultados similares fueron obtenidos para la celulosa y la madera de pino usando sulfato férrico [12]. En este trabajo se recurrió al uso de la técnica de análisis termogravimétrico para evaluar las características del proceso de descomposición térmica de la hemicelulosa pura y el efecto catalítico de los sulfatos de zinc y férrico sobre la pirólisis de este importante componente de la biomasa.

\section{Metodología}

\section{Materiales}

La hemicelulosa (xilano de madera de haya, >90\%) fue adquirida de Sigma-Aldrich y usada sin posterior purificación. Los catalizadores sulfato férrico (75 $\%)$ y sulfato de zinc (99 \%) fueron obtenidos de PanReac. Las mezclas de hemicelulosa/catalizador al $3 \%$ en peso de catalizador se prepararon por pesada usando cantidades adecuadas de cada uno de los componentes y se agitaron hasta lograr uniformidad. Posteriormente, fueron secadas isotérmicamente a $378 \mathrm{~K}$ durante 24 horas, para luego ser almacenadas en desecador.

\section{Análisis termogravimétrico (TGA)}

Los termogramas de la hemicelulosa con y sin catalizadores fueron obtenidos con una balanza termogravimétrica 2950 (TA Instruments). Con el objeto de reducir los efectos de transferencia de calor y masa, el rango de masa de las muestras se fijó entre 2 y $10 \mathrm{mg}$. Para el caso de la velocidad de calentamiento más alta, se usaron masas en el límite inferior de este rango [13]. Los ensayos se ejecutaron en atmósfera inerte de helio (grado alta pureza) con flujo constante de $100 \mathrm{ml} / \mathrm{min}$, a tres velocidades de calentamiento: $10 \mathrm{~K} / \mathrm{min}, 30 \mathrm{~K} / \mathrm{min}$ y $100 \mathrm{~K} / \mathrm{min}$.

\section{Cinética de la pirólisis}

Los datos termogravimétricos se ajustaron a tres modelos comúnmente usados en la literatura especializada: el método diferencial de Friedman con modelo de orden de reacción $n$; el método de Ozawa, Flynn y Wall (OFW) o isoconversional, y el modelo de distribución de energías de activación, DAEM [14-16].

Modelo de orden de reacción $\boldsymbol{n}$ : la ecuación de velocidad de descomposición de una sustancia sólida que se calienta a una velocidad constante $\boldsymbol{\beta}=\frac{d \boldsymbol{T}}{\boldsymbol{d} \boldsymbol{t}}$ puede escribirse de la siguiente manera [17]:

$$
\beta\left(\frac{d \alpha}{d T}\right)=A e \frac{-E a}{R T} f(\alpha)
$$

Donde $A$ es el factor preexponencial $\mathrm{o}$ de frecuencia; $E a$ la energía de activación; $R$, la constante universal de los gases; $T$, la temperatura absoluta; $f(\alpha)$, la función cinética que representa el modelo cinético, y $\alpha$, la conversión. Al aplicar la función logaritmo neperiano a la ecuación (1) y al asumir el modelo de reacción $f(\alpha)=(1-\alpha)^{n}$, típicamente usado en este tipo de procesos y análisis [14], se obtiene lo siguiente:

$$
\ln \left(\frac{d \alpha}{d T}\right)-n \ln (1-\alpha)=\ln \left(\frac{A}{\beta}\right)-\frac{E a}{R T}
$$

Si se grafica la parte izquierda de la ecuación (2) en función de $1 / T$, es posible obtener la energía de activación a partir de la pendiente y el factor preexponencial del intercepto con la ordenada.

Método isoconversional: En este método, la energía de activación se relaciona con la velocidad de calentamiento y la temperatura a una conversión constante [15, 13], así:

$$
\ln \beta=C_{1}-\frac{E a}{R T}
$$

Donde $C_{1}$ es una constante. La energía de activación puede calcularse a partir de la pendiente de $\ln \beta$ en función de $1 / T$. 
Modelo de distribución de energías de activación: Este método ha sido ampliamente descrito en [16-22]. El método agrupa conjuntos de reacciones paralelas y supone que las energías de activación de dichas reacciones se encuentran distribuidas según alguna distribución estadística, normalmente la función de distribución normal. El método transforma la integral de densidad para la velocidad de reacción en una suma que se va a evaluar numéricamente:

$x_{j}(t)=\frac{1}{2 \sqrt{\pi}} \int_{-\infty}^{\infty} \exp \left[\mu_{j} 2\right] \exp \left[0,75 \mu_{j} 2\right] X_{j}\left(t, \mu_{j}\right) d \mu_{j}$

Donde $x_{j}(t)$ es la fracción del material sin reaccionar en el tiempo; $X_{j}(t, E)$ la solución de la ecuación cinética de primer ordenpara una energía de activación dada; $\mu_{j}=2\left(E-E_{0 j}\right) /\left(\sqrt{2} \sigma_{j}\right)$, un cambio de variable que depende de la energía de activación promedio $E_{0 j}$ y de la desviación estándar de la distribución de energías de activación $\sigma_{j}$. La derivada de la conversión se calcula de la siguiente manera:

$$
\frac{d \alpha}{d t}^{\text {calc }}(t)=-\sum_{j=1}^{M} C j \frac{d x_{j}}{d t}
$$

El ajuste no lineal se logra minimizando por un método de búsqueda directo, codificado en Matlab®, la ecuación:

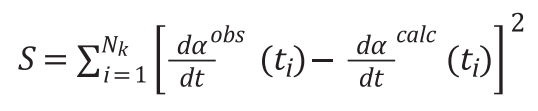

\section{Resultados y discusión}

La Figura 1 muestra los termogramas TG de la pirólisis de la hemicelulosa en presencia y ausencia de los catalizadores sulfato férrico y sulfato de zinc a las velocidades de calentamiento 10,30 y $100 \mathrm{~K} / \mathrm{min}$. El perfil del termograma, el rango de temperatura en el cual tiene lugar la reacción de pirólisis y la temperatura de máxima velocidad de descomposición para la hemicelulosa sin catalizadores es consistente con los resultados reportados en la literatura [23, 24]. Para la hemicelulosa en presencia y ausencia de estos catalizadores, se encontró que el porcentaje de pérdida de peso (en el mismo rango de temperatura) es mucho mayor que el reportado para otro componente principal de la biomasa, la lignina [23]. Se observa que a todas las velocidades de calentamiento estudiadas, el porcentaje de carbonizado de la pirólisis de los sistemas estudiados aumenta en el orden hemicelulosa pura, hemicelulosa con sulfato férrico, hemicelulosa con sulfato de zinc. Para la pirólisis de la hemicelulosa pura, el porcentaje de carbonizado no cambia al aumentar la velocidad de calentamiento de $10 \mathrm{~K} / \mathrm{min}$ a $100 \mathrm{~K} / \mathrm{min}$. En cambio, para la pirólisis de la muestra de hemicelulosa en presencia de sulfato férrico, el porcentaje de carbonizado máximo ocurre cuando la muestra se calienta a $30 \mathrm{~K} / \mathrm{min}$, y para la pirólisis de la muestra de hemicelulosa en presencia de sulfato de zinc el porcentaje de carbonizado mínimo se presenta a la velocidad de calentamiento $30 \mathrm{~K} / \mathrm{min}$. Estos resultados concuerdan con el efecto que estos catalizadores tienen sobre diferentes biomasas; sin embargo, en estudios anteriores, los resultados se han interpretado en términos de los componentes mayoritarios, lignina y celulosa. Nuestros resultados muestran que también hay una contribución de la hemicelulosa al aumento de carbonizado en presencia de estos catalizadores [25].

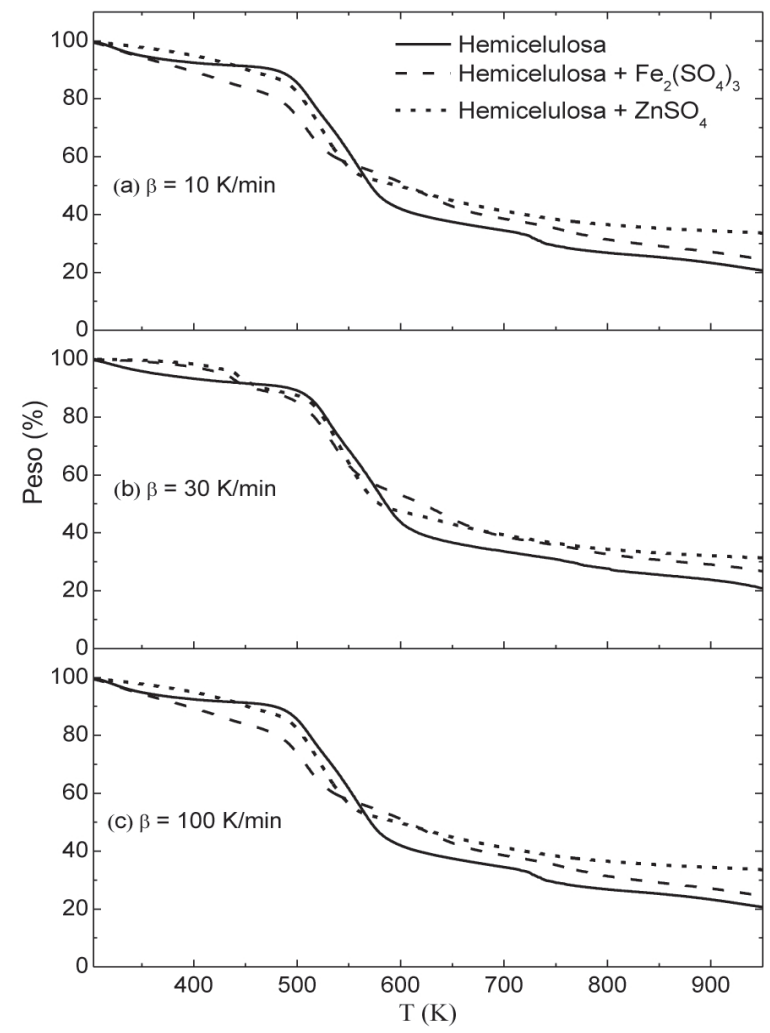

Figura 1. Termogramas TG de la pirólisis de la hemicelulosa pura y en mezcla con los catalizadores sulfato férrico y sulfato de zinc. Tres muestras de cada uno de estos sistemas de calentaron respectivamente a 10,30 y $100 \mathrm{~K} / \mathrm{min}$. 
La Figura 2 muestra las curvas DTG calculadas de los termogramas TG presentados en la Figura 1. En términos generales, se observan dos eventos principales: el primero corresponde a la liberación de humedad, y el segundo, en un rango de temperatura entre 413 a $723 \mathrm{~K}$, al proceso de pirólisis. La cota superior del rango de temperatura en el cual ocurre la reacción de pirólisis de la hemicelulosa pura es aproximadamente $623 \mathrm{~K}$, pero en presencia de los sulfatos estudiados, este se extiende hasta $723 \mathrm{~K}$. El perfil de estos termogramas muestra una fuerte dependencia con la adición de los catalizadores, incluyendo separación de los dos picos observados en el termograma de la pirólisis de la hemicelulosa pura y la aparición de nuevos picos. La hemicelulosa pura presenta, dentro del rango de temperatura de interés, un evento térmico bien definido con dos picos que se solapan. Al aumentar la velocidad de calentamiento, cambia la altura relativa de los picos, esto es, la velocidad máxima de descomposición de la muestra para cada evento. Específicamente, a la velocidad de calentamiento $10 \mathrm{~K} / \mathrm{min}$, el primer pico presenta una altura menor que el segundo pico; a $30 \mathrm{~K} / \mathrm{min}$ los dos picos presentan la misma altura, y a $100 \mathrm{~K} / \mathrm{min}$, la altura del segundo pico es mayor que la del primero. Esto sugiere que la velocidad de calentamiento tiene un efecto significativo sobre uno o ambos eventos cinéticos asociados a los picos observados en el rango del evento principal de pirólisis para la hemicelulosa pura.

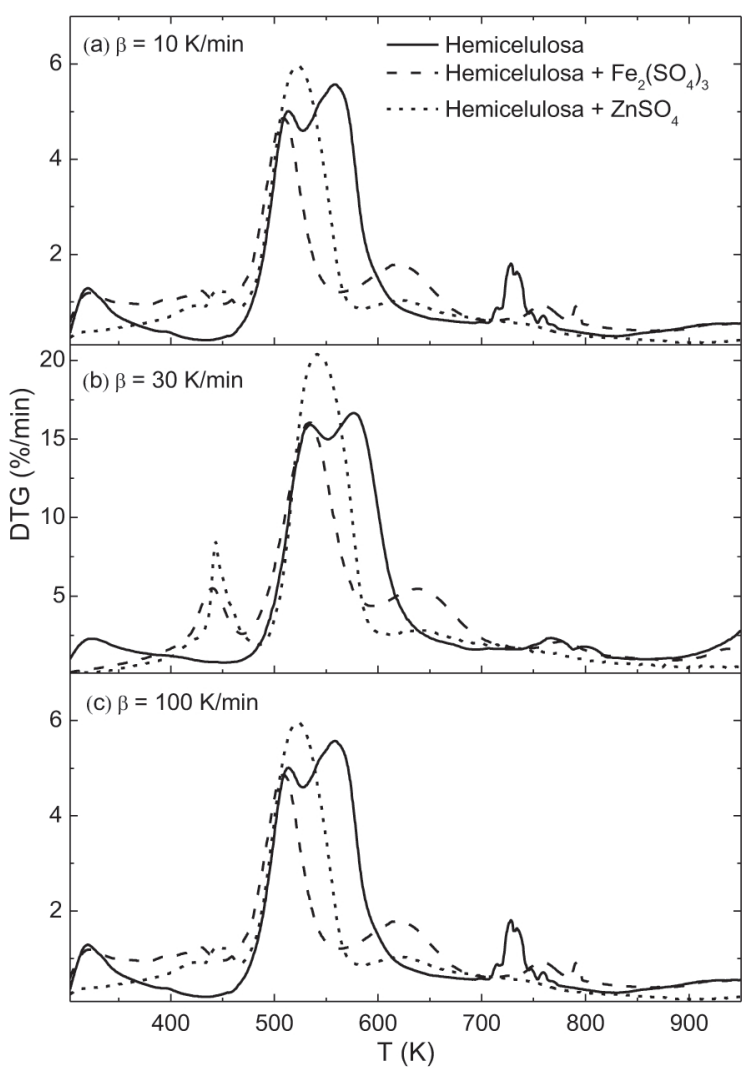

Figura 2. Curvas DTG calculadas a partir de la derivada con el tiempo de los termogramas TG mostrados en la Figura 1.

De acuerdo con los resultados mostrados en la Figura 2, a todas las velocidades de calentamiento los termogramas de la pirólisis de la hemicelulosa en presencia de sulfato férrico presentan tres picos claramente definidos, en contraste con los dos picos solapados que se observan en el termograma de la pirólisis de la hemicelulosa pura: un primer pico que se evidencia a velocidades de calentamiento de 30 y $100 \mathrm{~K} /$ min y que inicia alrededor de $413 \mathrm{~K}$; un segundo evento con una velocidad máxima de descomposición y temperatura de pico similar al primer pico de la pirólisis de la hemicelulosa pura, 
el cual se observa para todas las velocidades de calentamiento; y un tercer pico que se desplaza a temperaturas más altas con respecto al segundo pico de la pirólisis de la hemicelulosa pura: para la velocidad de calentamiento $10 \mathrm{~K} / \mathrm{min}$ este desplazamiento es de $40 \mathrm{~K}$, y para las muestras que se calientan a 30 y $100 \mathrm{~K} / \mathrm{min}$ es de $70 \mathrm{~K}$.

Por otra parte, el sulfato de zinc cambia considerablemente el perfil del termograma de la pirólisis de la hemicelulosa dentro del rango de temperatura de interés, mostrando un primer pico que inicia alrededor de $413 \mathrm{~K}$, el cual contrasta con el primer pico que se presenta para la pirólisis de la hemicelulosa pura que inicia alrededor de 473 $\mathrm{K}$; adicionalmente, se observa un segundo pico con una temperatura de máxima velocidad de descomposición, cuyo valor se encuentra entre las temperaturas de los dos picos que presentan las muestras de hemicelulosa pura. Este segundo pico de la pirólisis catalítica de la hemicelulosa con sulfato de zinc exhibe la mayor velocidad de descomposición entre todas las muestras a todas las velocidades de calentamiento usadas en este trabajo. El cambio de la forma de los perfiles ha sido reportado para la celulosa y otras sales usadas como catalizadores [26].

La influencia que tienen los catalizadores sobre la reacción de pirólisis de la hemicelulosa se confirmó a través del análisis de varianza (ANOVA) del diseño experimental. Se tomaron como variables manipuladas el tipo de catalizador y la velocidad de calentamiento, y como variable de respuesta la temperatura de pico del primer pico dentro del rango de temperatura de pirólisis de las muestras. El ANOVA mostró que las dos variables manipuladas tienen un efecto significativo sobre la temperatura de pico, con un nivel de confianza del $95 \%$ (véase tabla 1). Se encontraron resultados similares con otras sales inorgánicas a concentraciones tan bajas como $0,01 \%$ en peso, para otro de los componentes de la biomasa, la celulosa [26]. Al ser el xilano y la celulosa ambos polímeros de azúcares, los efectos significativos de las sales agregadas en el estudio sobre los parámetros de pirólisis no resultan sorprendentes.

Tabla 1. Análisis de varianza del efecto de las variables de estudio sobre la temperatura del primer pico de pirólisis de la hemicelulosa en ausencia y presencia de sulfato férrico y sulfato de zinc.

\begin{tabular}{ccccc}
\hline Fuente/Efectos principales & Catalizador & $\begin{array}{c}\text { Velocidad de } \\
\text { calentamiento }(\boldsymbol{\beta})\end{array}$ & Residuales & $\begin{array}{c}\text { Total } \\
\text { (corregidos) }\end{array}$ \\
\hline Suma de cuadrados & 244,92 & 1310,21 & 298,014 & 1584,93 \\
GL & 2 & 2 & 4 & 8 \\
Cuadrado medio & 122,46 & 655,106 & 745,035 & \\
Razón - F & 16,44 & 87,93 & & \\
Valor - P & 0,0118 & 0,0005 & & \\
\hline
\end{tabular}

\section{Modelos cinéticos}

Modelo de orden de reacción $\mathrm{n}$ : A pesar de la aparente complejidad de los termogramas, los datos experimentales se ajustaron bien a este modelo (figura 3). Para el caso de la pirólisis de la hemicelulosa pura, se observó un orden de reacción 2 , independientemente de la velocidad de calentamiento (tabla 2). La presencia del sulfato férrico como catalizador cambia significativamente el orden de reacción de la pirólisis de la hemicelulosa a valores altos, poco realistas desde el punto de vista cinético y que varían considerablemente con la velocidad de calentamiento. Por otra parte, el sulfato de zinc disminuye el orden de reacción de la pirólisis de la hemicelulosa a 1,5. Este valor es constante a todas las velocidades de calentamiento estudiadas. El valor promedio de la energía de activación de la pirólisis de hemicelulosa pura obtenido usando con este modelo es $100 \pm 5 \mathrm{~kJ} /$ $\mathrm{mol}$, el cual está incluido en el rango de los valores reportados en la literatura $(95-267 \mathrm{~kJ} / \mathrm{mol})$ [2729]. Para el caso de la pirólisis de la hemicelulosa usando como catalizador el sulfato de zinc, el valor de la energía de activación obtenido es $127 \pm 8 \mathrm{~kJ} /$ mol. En términos generales, la aplicación de este modelo a los datos experimentales sugiere un cambio de mecanismo de reacción en presencia de los catalizadores estudiados. Resultados similares fueron obtenidos para la celulosa al agregar sales como el $\mathrm{ZnCl}_{2}$ [26]. 
Tabla 2. Resultados del ajuste de los datos cinéticos de la pirólisis de la hemicelulosa pura y en mezcla con los catalizadores sulfato férrico y sulfato de zinc al modelo de orden de reacción $n$.

\begin{tabular}{cccc}
\hline Sistema & $\boldsymbol{\beta}(\boldsymbol{K} / \mathbf{m i n})$ & $\boldsymbol{f}(\boldsymbol{\alpha})$ & $\boldsymbol{R}^{\mathbf{2}}$ \\
\hline Hemicelulosa & 10 & $(1-\alpha)^{2,1}$ & 0,989 \\
& 30 & $(1-\alpha)^{1,9}$ & 0,972 \\
& 100 & $(1-\alpha)^{2,1}$ & 0,962 \\
\hline \multirow{2}{*}{ Hemicelulosa $+\mathrm{Fe}_{2}\left(\mathrm{SO}_{4}\right)_{3}$} & 10 & $(1-\alpha)^{13}$ & 0,939 \\
& 30 & $(1-\alpha)^{6,4}$ & 0,949 \\
& 100 & $(1-\alpha)^{5,9}$ & 0,96 \\
\hline & 10 & $(1-\alpha)^{1,7}$ & 0,998 \\
Hemicelulosa $+\mathrm{ZnSO}_{4}$ & 30 & $(1-\alpha)^{1,3}$ & 0,998 \\
& 100 & $(1-\alpha)^{1,5}$ & 0,998
\end{tabular}
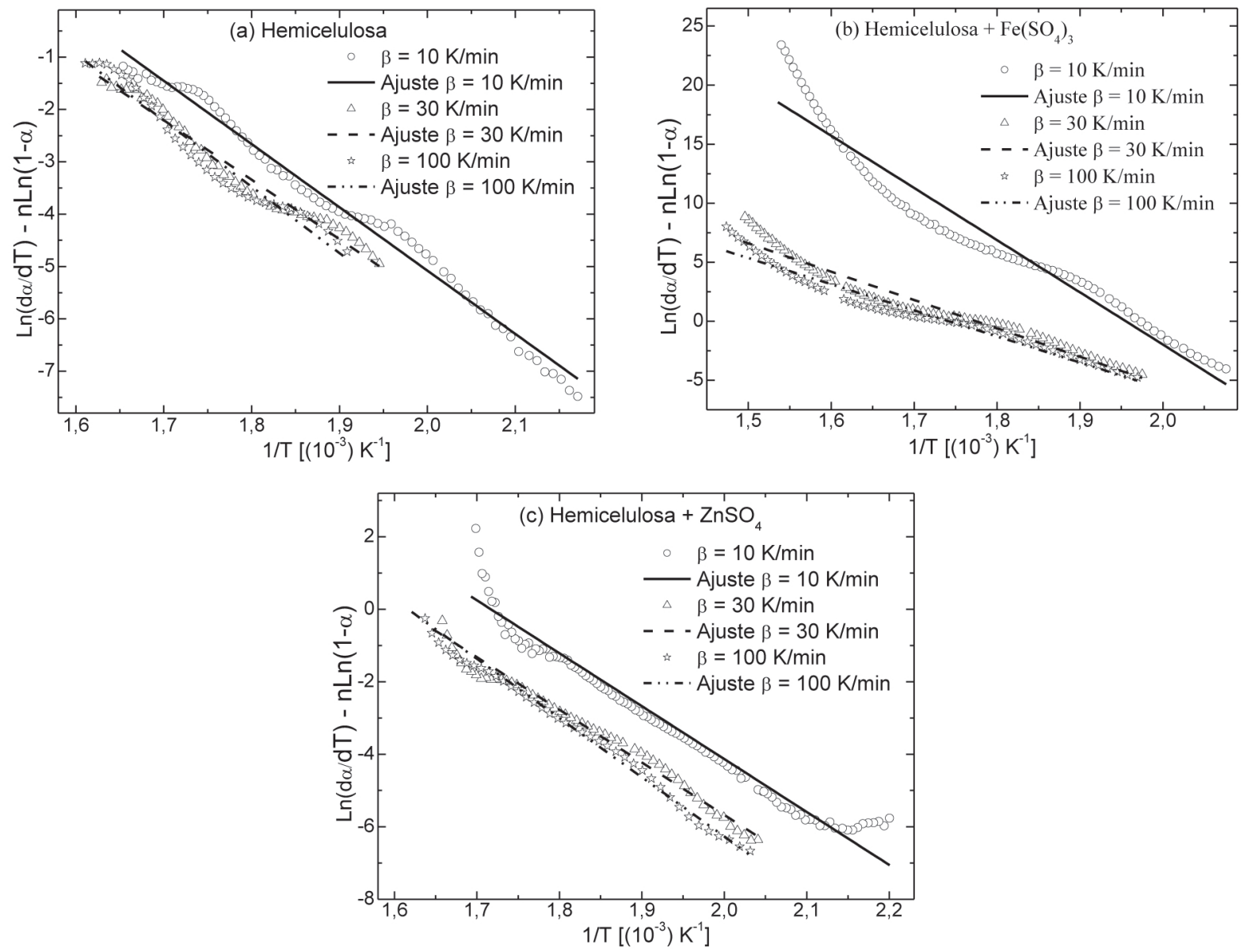

Figura 3. Ajuste al modelo de orden de reacción $n$ de la hemicelulosa pura y en mezcla con los catalizadores sulfato férrico y sulfato de zinc. 
Modelo isoconversional: los resultados del ajuste de los datos experimentales de la pirólisis de la hemicelulosa en presencia y ausencia de los catalizadores estudiados se muestran en la figura 4. Este modelo presentó un buen ajuste para la pirólisis de la hemicelulosa pura, con valores de coeficiente de correlación superiores a 0,9. Sin embargo, el modelo no ajusta bien a la reacción de pirólisis de la hemicelulosa catalizada por los sulfatos de estudio. Esto es de esperarse debido a los múltiples picos y a la separación que entre estos se presenta en el rango de temperatura del evento de interés, cuando la pirólisis de la hemicelulosa ocurre en presencia de los sulfatos estudiados. El valor de la energía de activación para la pirólisis de la hemicelulosa pura obtenida con este modelo es $272+29 \mathrm{~kJ} / \mathrm{mol}$, el cual es consistente con los valores reportados en la literatura [27-29]. Si bien el valor de la energía de activación para la pirólisis de la hemicelulosa pura está incluido en el rango reportado en la literatura, difiere considerablemente del valor obtenido para la misma reacción con el modelo de orden de reacción $n$. En este caso, el amplio rango de valores para la energía de activación de la pirólisis de la hemicelulosa reportado en este estudio y en la literatura puede atribuirse también a la fuente de donde es extraída, la naturaleza compleja de la molécula y las suposiciones hechas en los modelos de ajuste cinético [30].
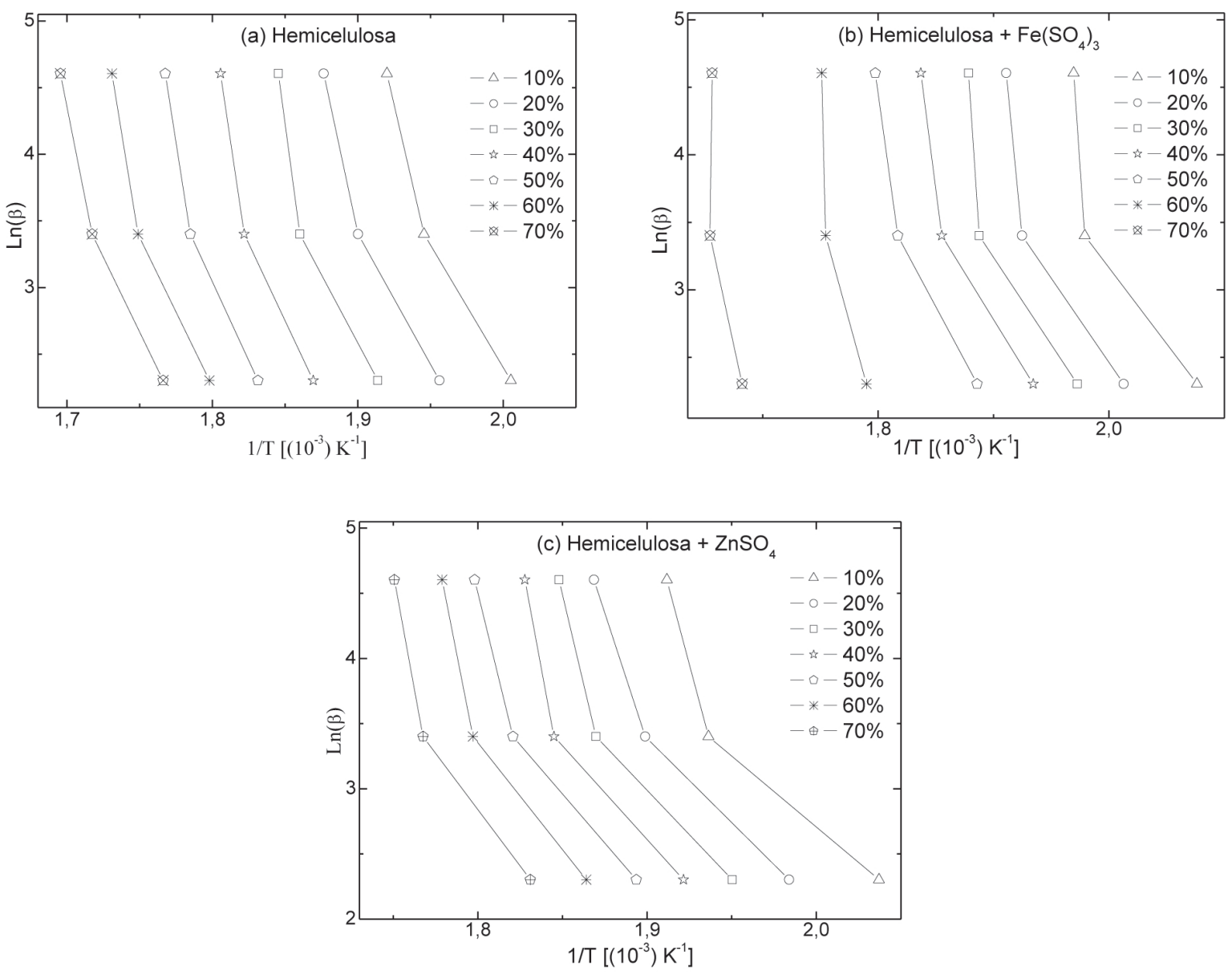

Figura 4. Ajuste de los datos de la pirólisis de hemicelulosa pura y en mezcla con los catalizadores objeto de estudio al método isoconversional. 
DAEM: El ajuste de los datos experimentales de la pirólisis de la hemicelulosa pura a velocidades de calentamiento 10,30 y $100 \mathrm{~K} / \mathrm{min}$ al modelo DAEM se muestra en la Figura 5 y los parámetros resultados del ajuste en la tabla 3 . Fueron necesarios 3 conjuntos de reacciones DAEM para describir el comportamiento de la pirólisis de la hemicelulosa en ausencia y presencia de los catalizadores, como se muestra en las Figuras 5 y 6. Cuando se comparan con los parámetros de la pirólisis de la hemicelulosa pura, los resultados del ajuste a este modelo muestran cambios en los parámetros cinéticos del segundo y tercer conjunto de reacciones DAEM, tanto para la pirólisis de la hemicelulosa catalizada con sulfato férrico como para la reacción catalizada por sulfato de zinc.
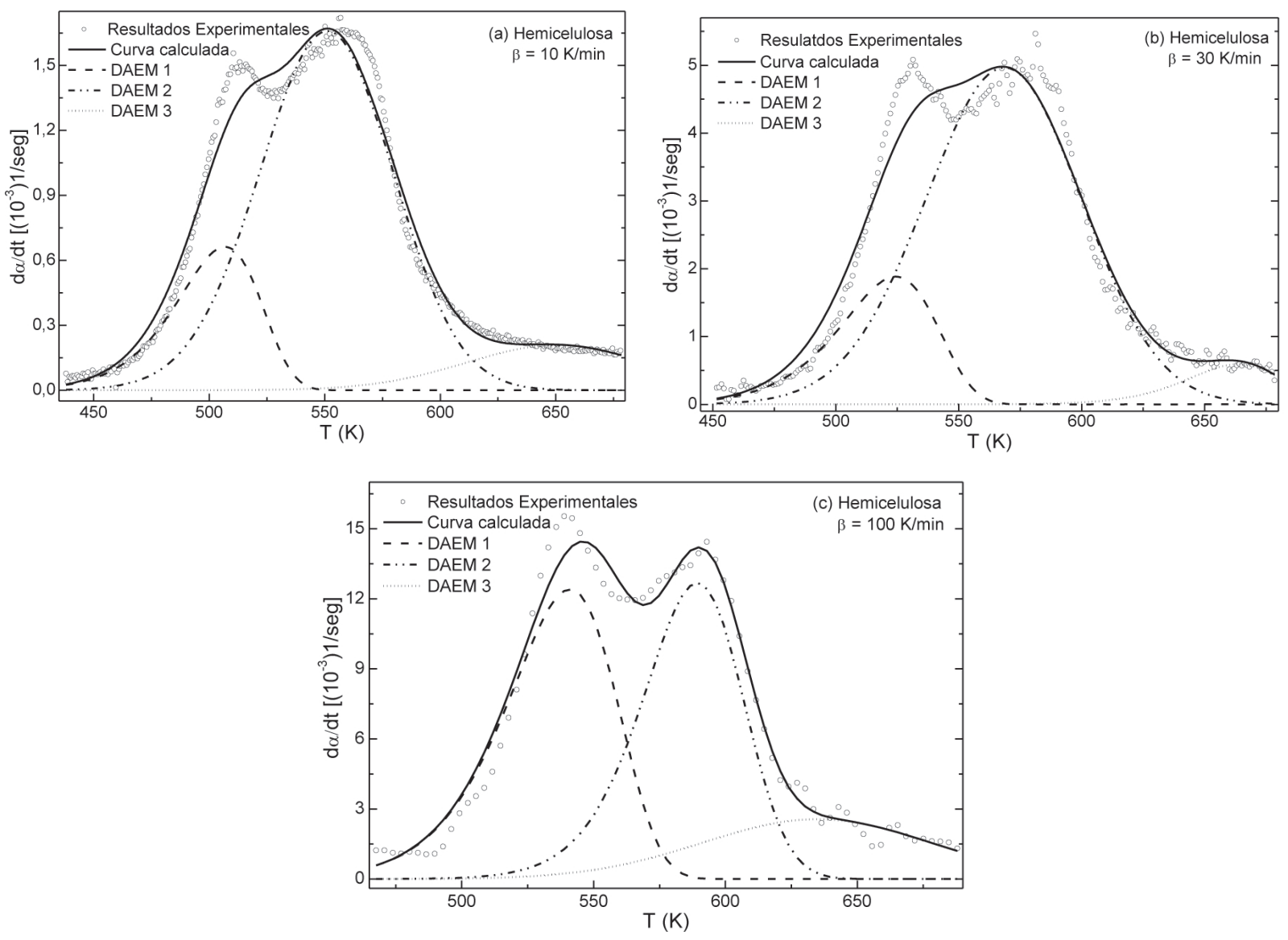

Figura 5. Ajuste del modelo DAEM con diferentes números de conjuntos de reacciones para la pirólisis de la hemicelulosa.

Teniendo en cuenta el esquema de pirólisis de hemicelulosa propuesto por [23], los catalizadores estudiados afectan en forma diferente cada uno de los pasos de este proceso. Ambos catalizadores aceleran fuertemente el proceso de deshidratación/fragmentación inicial de la hemicelulosa, y lo desplazan a temperaturas inferiores en comparación con la hemicelulosa pura; la reacción de depolimerización también es catalizada por ambas sales, pero el aumento en la velocidad no es tan grande como para el caso de la reacción de fragmentación, y el corrimiento del pico correspondiente a esta reacción hacia temperaturas inferiores es mucho menor que en el caso de la fragmentación/deshidratación. Estos efectos se ven reflejados en los cambios en los valores de los parámetros de las reacciones según los diferentes modelos utilizados en este trabajo. En el caso del sulfato férrico, se observa un tercer evento dentro del rango de interés que podría corresponder a descomposiciones adicionales del carbonizado durante su proceso de formación, que son catalizadas por esta sal, y cuya velocidad de reacción es tan baja en la hemicelulosa en ausencia de este catalizador que no es apreciable en los experimentos termogravimétricos. 
Tabla 3 Parámetros del modelo DAEM aplicado a la pirolisis de la hemicelulosa con y sin catalizadores.

\begin{tabular}{|c|c|c|c|}
\hline Parámetro & Hemicelulosa & Hemicelulosa $+\mathrm{Fe}_{2}\left(\mathrm{SO}_{4}\right)_{3}$ & Hemicelulosa $+\mathrm{ZnSO}_{4}$ \\
\hline \multicolumn{4}{|c|}{$\beta=10 \mathrm{~K} / \mathrm{min}$} \\
\hline $\mathrm{C}_{1}$ & 0,1882 & 0,1882 & 0,2188 \\
\hline$A_{1}\left(s^{-1}\right)$ & $4,36 \mathrm{E}+09$ & $3,30 E+09$ & $3,41 E+09$ \\
\hline $\mathrm{E}_{01}(\mathrm{~kJ} / \mathrm{mol})$ & 113 & 112 & 112 \\
\hline $\boldsymbol{\sigma}_{1}(\mathrm{~kJ} / \mathrm{mol})$ & 0,146 & 0,15 & 0,056 \\
\hline $\mathrm{c}_{2}$ & 0,7316 & 0,3538 & 0,7063 \\
\hline$A_{2}\left(s^{-1}\right)$ & $1,72 \mathrm{E}+15$ & $4,96 \mathrm{E}+16$ & $9,87 \mathrm{E}+15$ \\
\hline $\mathrm{E}_{02}(\mathrm{~kJ} / \mathrm{mol})$ & 183 & 183 & 183 \\
\hline $\boldsymbol{\sigma}_{2}(\mathrm{~kJ} / \mathrm{mol})$ & 8,279 & 7,575 & 5,335 \\
\hline $\mathrm{c}_{3}$ & 0,125 & 0,47 & 0,297 \\
\hline$A_{3}\left(s^{-1}\right)$ & $6,41 \mathrm{E}+13$ & $3,71 \mathrm{E}+14$ & $2,70 \mathrm{E}+14$ \\
\hline $\mathrm{E}_{03}(\mathrm{~kJ} / \mathrm{mol})$ & 199 & 198 & 198 \\
\hline$\sigma_{3}(\mathrm{~kJ} / \mathrm{mol})$ & 10,7 & 15,3 & 10,4 \\
\hline \multicolumn{4}{|c|}{$\beta=30 \mathrm{~K} / \mathrm{min}$} \\
\hline $\mathrm{c}_{1}$ & 0,1882 & 0,2876 & 0,3496 \\
\hline$A_{1}\left(s^{-1}\right)$ & $4,62 E+09$ & $2,45 E+09$ & $6,33 E+08$ \\
\hline $\mathrm{E}_{01}(\mathrm{~kJ} / \mathrm{mol})$ & 113 & 112 & 112 \\
\hline $\boldsymbol{\sigma}_{1}(\mathrm{~kJ} / \mathrm{mol})$ & 0,146 & 0,360 & 0,025 \\
\hline $\mathrm{C}_{2}$ & 0,7784 & 0,2594 & 0,6261 \\
\hline$A_{2}\left(s^{-1}\right)$ & $1,47 \mathrm{E}+15$ & $2,13 E+16$ & $1,43 E+16$ \\
\hline $\mathrm{E}_{02}(\mathrm{~kJ} / \mathrm{mol})$ & 183 & 183 & 183 \\
\hline $\boldsymbol{\sigma}_{2}(\mathrm{~kJ} / \mathrm{mol})$ & 8,96 & 7,12 & 5,20 \\
\hline $\mathrm{c}_{3}$ & 0,0594 & 0,4718 & 0,3523 \\
\hline$A_{3}\left(s^{-1}\right)$ & $1,84 \mathrm{E}+15$ & $4,80 \mathrm{E}+14$ & $3,65 E+14$ \\
\hline $\mathrm{E}_{03}(\mathrm{~kJ} / \mathrm{mol})$ & 214 & 198 & 198 \\
\hline$\sigma_{3}(\mathrm{~kJ} / \mathrm{mol})$ & 3,04 & 17,415 & 0,001 \\
\hline \multicolumn{4}{|c|}{$\beta=100 \mathrm{~K} / \mathrm{min}$} \\
\hline $\mathrm{c}_{1}$ & 0,4393 & 0,2749 & 0,0756 \\
\hline$A_{1}\left(s^{-1}\right)$ & $6,25 E+09$ & $4,74 \mathrm{E}+09$ & $9,90 \mathrm{E}+09$ \\
\hline $\mathrm{E}_{01}(\mathrm{~kJ} / \mathrm{mol})$ & 113 & 112 & 114 \\
\hline $\boldsymbol{\sigma}_{1}(\mathrm{~kJ} / \mathrm{mol})$ & 0,054 & 0,408 & 0,025 \\
\hline $\mathrm{C}_{2}$ & 0,4171 & 0,351 & 0,8994 \\
\hline$A_{2}\left(s^{-1}\right)$ & $1,33 \mathrm{E}+15$ & $3,76 \mathrm{E}+16$ & $1,17 \mathrm{E}+16$ \\
\hline $\mathrm{E}_{02}(\mathrm{~kJ} / \mathrm{mol})$ & 183 & 183 & 183 \\
\hline $\boldsymbol{\sigma}_{2}(\mathrm{~kJ} / \mathrm{mol})$ & 3,18 & 10,6 & 5,62 \\
\hline $\mathrm{c}_{3}$ & 0,1808 & 0,3949 & 0,2891 \\
\hline $\mathrm{A}_{3}\left(\mathrm{~s}^{-1}\right)$ & $1,63 E+15$ & $4,63 E+14$ & $3,70 \mathrm{E}+14$ \\
\hline $\mathrm{E}_{03}(\mathrm{~kJ} / \mathrm{mol})$ & 200 & 198 & 198 \\
\hline$\sigma_{3}(\mathrm{~kJ} / \mathrm{mol})$ & 12,3 & 15,0 & 6,17 \\
\hline
\end{tabular}


Los resultados obtenidos en esta investigación muestran un fuerte efecto catalítico de las sales sobre la pirólisis de la hemicelulosa. Esto se hizo evidente en el cambio de la forma de los termogramas y con el análisis cinético implementado con los tres modelos de ajuste cinético usados en esta investigación.
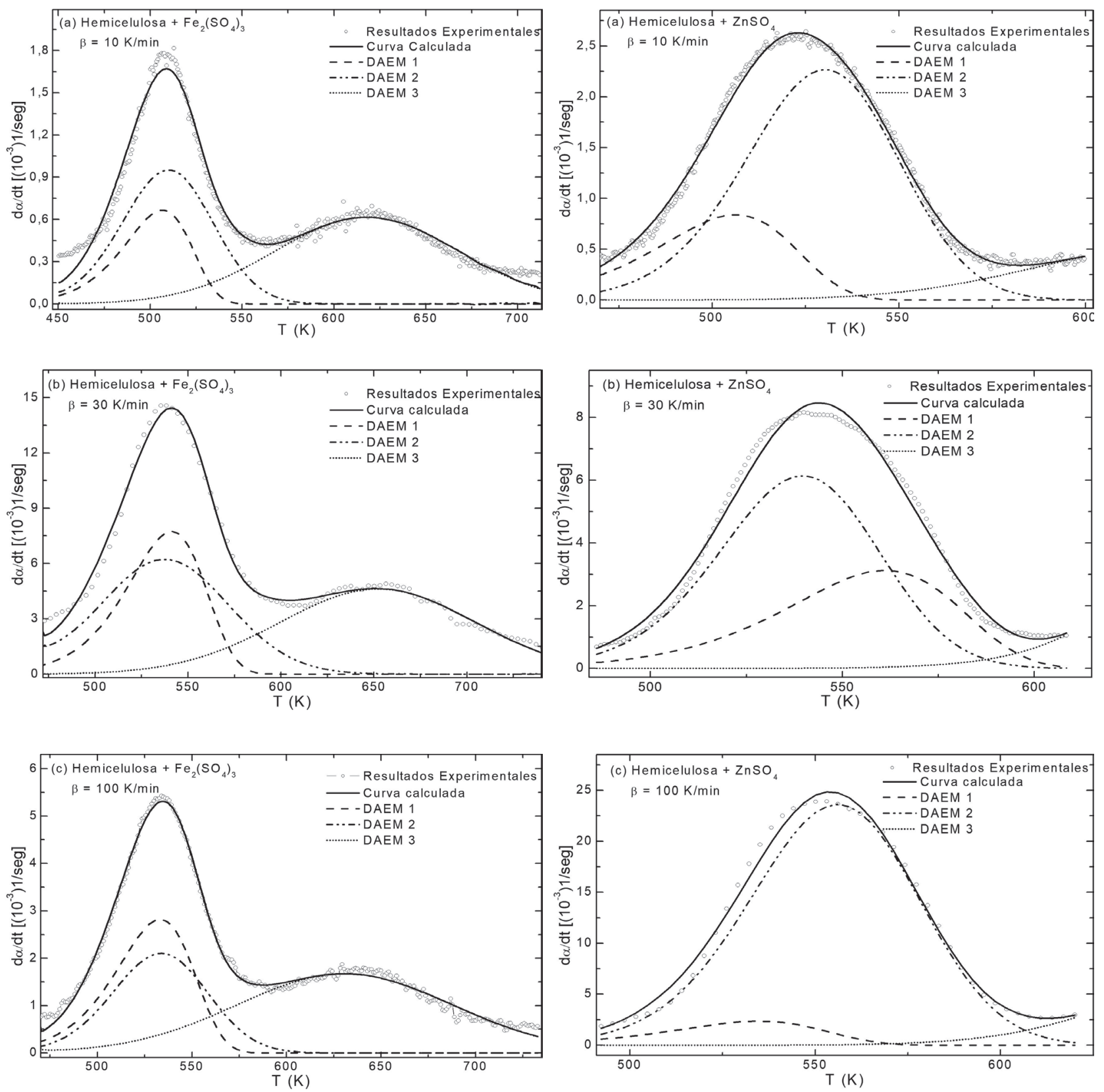

Figura 6. Ajustes de las curvas DTG de hemicelulosa con $\mathrm{Fe}_{2}\left(\mathrm{SO}_{4}\right)_{3}$ y hemicelulosa con $\mathrm{ZnSO}_{4}$ a tres velocidades de calentamiento, usando DAEM. 


\section{Conclusiones}

Usando la técnica de caracterización análisis termogravimétrico, se estudió el efecto de los sulfatos férrico y de zinc sobre la pirólisis de la hemicelulosa, componente esencial de la biomasa lignocelulósica. Los resultados fueron ajustados a tres modelos cinéticos: modelo de orden de reacción $n$, modelo isoconversional y DAEM. La pirólisis de la hemicelulosa pura se ajustó bien a los tres modelos; sin embargo, la pirólisis de la hemicelulosa catalizada por el sulfato férrico sólo ajustó al modelo DAEM y la pirólisis de la hemicelulosa catalizada por sulfato de zinc ajustó al modelo de orden de reacción $n$ y al modelo DAEM con tres conjuntos de reacciones. En términos generales, se encontró un fuerte efecto catalítico de estas sales sobre la pirólisis de la hemicelulosa. Esto se hizo evidente en el cambio de perfil de los termogramas y en el análisis cinético implementado con los indicados modelos. La comparación de los resultados obtenidos con el modelo DAEM y el modelo de descomposición propuesto en la literatura sugiere que los sulfatos, objeto de la presente investigación, catalizan las reacciones de deshidratación/fragmentación y depolimerización de la hemicelulosa, lo que disminuye la temperatura de la pirólisis de este componente y favorece potencialmente, por esta ruta termoquímica, el balance energético de la producción de biocombustibles a partir de biomasa.

\section{Referencias bibliográficas}

[1] Bridgwater T. Biomass for energy. J. Sci. Food Agric. 2006;86(12):1755-68.

[2] Sonobe T, Worasuwannarak N. Kinetic analyses of biomass pyrolysis using the distributed activation energy model. Fuel. 2008;87(3):414-21.

[3] Wang S, Guo X, Wang K, Luo Z. Influence of the interaction of components on the pyrolysis behavior of biomass. J. Anal. Appl. Pyrolysis. 2011;91(1):183-9.

[4] Worasuwannarak N, Sonobe T, Tanthapanichakoon W. Pyrolysis behaviors of rice straw, rice husk, and corncob by TG-MS technique. J. Anal. Appl. Pyrolysis. 2007;78(2):265-71.

[5] Granada E, Eguía P, Comesaña J, Patiño D, Porteiro J, Miguez J. Devolatilization behaviour and pyrolysis kinetic modelling of Spanish biomass fuels. J. Therm. Anal. Calorim.
2013;113(2):569-78.

[6] Gani A, Naruse I. Effect of cellulose and lignin content on pyrolysis and combustion characteristics for several types of biomass. Renew. Energy. 2007;32(4):649-61.

[7] Yang H, Yan R, Chen H, Lee DH ,Zheng C. Characteristics of hemicellulose, cellulose and lignin pyrolysis. Fuel. 2007;86(12):1781-8.

[8] Lv D, Xu M, Liu X, Zhan Z, Li Z, Yao H. Effect of cellulose, lignin, alkali and alkaline earth metallic species on biomass pyrolysis and gasification. Fuel Process. Technol. 2010;91(8):903-9.

[9] Khelfa A, Bensakhria A, Weber J. Investigations into the pyrolytic behaviour of birch wood and its main components: primary degradation mechanisms, additivity and metallic salt effects. J. Anal. Appl. Pyrolysis. 2013;101:11121.

[10]Zhang H, Xiao R, Jin B, Xiao G, Chen R. Biomass catalytic pyrolysis to produce olefins and aromatics with a physically mixed catalyst. Bioresour. Technol. 2013;140:256-62.

[11] Richards GN, Zheng G. Influence of metal ions and of salts on products from pyrolysis of wood: applications to thermochemical processing of newsprint and biomass. J. Anal. Appl. Pyrolysis. 1991;21(1-2):133-46.

[12] Dobele G, Rossinskaja G, Dizhbite T, Telysheva G, Meier D, Faix O. Application of catalysts for obtaining 1, 6-anhydrosaccharides from cellulose and wood by fast pyrolysis. J. Anal. Appl. Pyrolysis. 2005;74(1):401-05.

[13] Albis A, Ortiz E, Suárez A, Piñeres I. TG/MS study of the thermal devolatization of Copoazú peels (Theobroma grandiflorum). J. Therm. Anal. Calorim. 2014;115(1):275-83.

[14] Martín-Lara M, Blázquez G, Zamora M, Calero M. Kinetic modelling of torrefaction of olive tree pruning. Appl. Therm. Eng. 2017;113:1410-8.

[15] Açıkalın K. Pyrolytic characteristics and kinetics of pistachio shell by thermogravimetric analysis. J. Therm. Anal. Calorim. 2011;109(1):227-35.

[16] Abdelouahed L, Leveneur S, VernieresHassimi L, Balland L, Taouk B. Comparative investigation for the determination of kinetic parameters for biomass pyrolysis by thermogravimetric analysis. J. Therm. Anal. Calorim. 2017:1-13.

[17] Chen N, Ren J, Ye Z, Xu Q, Liu J, Sun S. Kinetics of coffee industrial residue pyrolysis using distributed activation energy model and components separation of bio-oil by 
sequencing temperature-raising pyrolysis. Bioresour. Technol. 2016;221:534-40.

[18]Cheng Z, Wu W, Ji P, Zhou X, Liu R, Cai J. Applicability of Fraser-Suzuki function in kinetic analysis of DAEM processes and lignocellulosic biomass pyrolysis processes. J. Therm. Anal. Calorim. 2015;119(2):1429-38.

[19]Donskoi E, McElwain D. Optimization of coal pyrolysis modeling. Combustion and Flame. 2000;122(3):359-67.

[20] Janković B. The pyrolysis process of wood biomass samples under isothermal experimental conditions-energy density considerations: application of the distributed apparent activation energy model with a mixture of distribution functions. Cellulose. 2014;21(4):2285-314.

[21]Várhegyi G. Aims and methods in nonisothermal reaction kinetics. J. Anal. Appl. Pyrolysis. 2007;79(1):278-88.

[22] Várhegyi G, Szabó P, Antal MJ. Kinetics of charcoal devolatilization. Energy \& fuels. 2002;16(3):724-31.

[23] Collard F-X, Blin J. A review on pyrolysis of biomass constituents: Mechanisms and composition of the products obtained from the conversion of cellulose, hemicelluloses and lignin. Renew. Sustain. Energy Rev. 2014;38:594-608.
[24]Dorez G, Ferry L, Sonnier R, Taguet A, Lopez-Cuesta J-M. Effect of cellulose, hemicellulose and lignin contents on pyrolysis and combustion of natural fibers. J. Anal. Appl. Pyrolysis. 2014;107:323-31.

[25] Zhou X, Li W, Mabon R, Broadbelt LJ. A critical review on hemicellulose pyrolysis. Energy Technology. 2017;5(1):52-79.

[26] Williams PT, Horne PA. The role of metal salts in the pyrolysis of biomass. Renew. Energy. 1994;4(1):1-13.

[27] Jakab E. Analytical techniques as a tool to understand the reaction mechanism. En: Recent advances in thermo-chemical conversion of biomass. EEUU: Elsevier; 2015. p. 75-108.

[28] Saddawi A, Jones J, Williams A, Wojtowicz $M$. Kinetics of the thermal decomposition of biomass. Energy \& fuels. 2009;24(2):1274-82.

[29]Zhang J, Chen T, Wu J, Wu J. MultiGaussian-DAEM-reaction model for thermal decompositions of cellulose, hemicellulose and lignin: comparison of $\mathrm{N} 2$ and $\mathrm{CO} 2$ atmosphere. Bioresour. Technol. 2014;166:8795.

[30]White JE, Catallo WJ, Legendre BL. Biomass pyrolysis kinetics: a comparative critical review with relevant agricultural residue case studies. J. Anal. Appl. Pyrolysis. 2011;91(1):1-33. 